Bull. Korean Math. Soc. 52 (2015), No. 1, pp. 287-299

http://dx.doi.org/10.4134/BKMS.2015.52.1.287

\title{
SUFFICIENT CONDITION FOR THE EXISTENCE OF THREE DISJOINT THETA GRAPHS
}

\author{
Yunshu Gao And Ding Ma
}

\begin{abstract}
A theta graph is the union of three internally disjoint paths that have the same two distinct end vertices. We show that every graph of order $n \geq 12$ and size at least $\left\lfloor\frac{11 n-18}{2}\right\rfloor$ contains three disjoint theta graphs. As a corollary, every graph of order $n \geq 12$ and size at least $\left\lfloor\frac{11 n-18}{2}\right\rfloor$ contains three disjoint cycles of even length.
\end{abstract}

\section{Terminology and introduction}

In this paper, we only consider finite undirected graphs, without loops or multiple edges. We use [1] for the notation and terminology not defined here. A theta graph is the union of three internally disjoint paths that have the same two distinct end vertices. Let $n$ be a positive integer, let $K_{n}$ denote the complete graph of order $n$ and $K_{4}^{-}$be the graph obtained by removing exactly one edge from $K_{4}$. For a graph $G$, we denote its vertex set, edge set, minimum degree by $V(G), E(G)$ and $\delta(G)$, respectively. The order and size of a graph $G$, are defined by $|V(G)|$ and $|E(G)|$, respectively. A set of subgraphs is said to be vertex-disjoint or independent, if no two of them have any common vertex in $G$, and we use disjoint to stand for vertex-disjoint throughout this paper. If $u$ is a vertex of $G$ and $H$ is either a subgraph of $G$ or a subset of $V(G)$, we define $N_{H}(u)$ to be the set of neighbors of $u$ contained in $H$, and $d_{H}(u)=\left|N_{H}(u)\right|$. For a subset $U$ of $V(G), G[U]$ denotes the subgraph of $G$ induced by $U$. In particular, we often use $[U]$ to stand for $G[U]$. If $S$ is a set of subgraphs of $G$, we write $G \supseteq S$, it means that $S$ is isomorphic to a subgraph of $G$, in particular, we use $m S$ to represent a set of $m$ vertex-disjoint copies of $S$. When $S=\left\{x_{1}, x_{2}, \ldots, x_{t}\right\}$, we may also use $\left[x_{1}, x_{2}, \ldots, x_{t}\right]$ to denote $\left[\left\{x_{1}, x_{2}, \ldots, x_{t}\right\}\right]$. Let $V_{1}, V_{2}$ be two disjoint subsets or subgraphs of $G$, we use $E\left(V_{1}, V_{2}\right)$ to denote the set of edges in $G$ with one end-vertex in $V_{1}$, while the other in $V_{2}$, for simplicity, let $E\left(x, V_{2}\right)$ stand for $E\left(\{x\}, V_{2}\right), E\left(V_{1}, x\right)$

Received February 5, 2014; Revised July 10, 2014.

2010 Mathematics Subject Classification. 05C35, 05C70.

Key words and phrases. disjoint theta graphs, sufficient condition, minimum degree.

Supported by the National Natural Science Foundation of China (Grant No. 11161035) and Ningxia Ziran (Grant No.NZ1153). 
for $E\left(V_{1},\{x\}\right)$, respectively. A path of order $n$ is denoted by $P_{n}$. Throughout this paper, we consider that any cycle has a fixed orientation. Let $C$ be a cycle of $G$. For $x, y \in V(C)$, we denote by $\vec{C}[x, y]$ the path from $x$ to $y$ on $\vec{C}$. A vertex $u$ is called a leaf of $G$ if $d_{G}(u)=1$.

Corrádi and Hajnal [3] proved the following well-known result on the existence of vertex-disjoint cycles in graphs.

Theorem $1.1([3])$. Let $k$ be a positive integer and $G$ be a graph with order $n \geq 3 k$. If $\delta(G) \geq 2 k$, then $G$ contains $k$ disjoint cycles.

Later, Wang [10] and independently Enomoto [5] proved a result stronger than Theorem 1.1 as follows.

Theorem 1.2 ([10]). Let $k$ be a positive integer and $G$ be a graph with order $n \geq 3 k$. Suppose for any pair of nonadjacent $u$ and $v$ in $G, d_{G}(u)+d_{G}(v) \geq$ $4 k-1$, then $G$ contains $k$ disjoint cycles.

Given a cycle $C$ of a graph $G$, a chord of $C$ is an edge of $G-E(C)$ which joins two vertices of $C$. A cycle is called a chorded cycle if it has at least one chord. A theta graph is the union of three internally disjoint paths that have the same two distinct end vertices. A chorded cycle is a simple example of a theta graph but, in general a theta graph need not be a chorded cycle. It is obvious that $K_{4}^{-}$is the theta graph with minimum order and every theta graph contains a cycle of even length. Pósa [9] proved that any graph with minimum degree at least three contains a chorded cycle. Motivated by these results, Finkel et al. [6] and Chiba et al. [3] obtained the following results analogous to Theorem 1.2, respectively.

Theorem 1.3 ([6]). If $G$ is a graph of order $n \geq 4 k$ and $\delta(G) \geq 3 k$, then $G$ contains $k$ disjoint chorded cycles.

Theorem 1.4 ([3]). Let $r, s$ be two nonnegative integers and let $G$ be a graph with order $n \geq 3 r+4 s$. Suppose for any pair of nonadjacent $u$ and $v$ in $G$, $d_{G}(u)+d_{G}(v) \geq 4 r+6 s-1$, then $G$ contains $r+s$ disjoint cycles such that $s$ of them are chorded cycles.

Kawarabayashi [8] considered the minimum degree to ensure the existence of disjoint copies of $K_{4}^{-}$in a general graph $G$, which can be seen as a specified version of disjoint chorded cycles.

Theorem $1.5([8])$. Let $k$ be a positive integer and $G$ be a graph with order $n \geq 4 k$. If $\delta(G) \geq\left\lceil\frac{n+k}{2}\right\rceil$, then $G$ contains $k$ disjoint copies of $K_{4}^{-}$.

In this paper, we determine the edge number for a graph to contain three disjoint theta graphs. Our research is motivated by the conjecture put forward by Gao and Ji [7]. 
Conjecture $1.6([7])$. Let $k \geq 2$ be an integer. Every graph of order $n$ and size at least $f(n, k)+1$ contains $k$ disjoint theta graphs, when

$f(n, k)=\max \left\{\left(\begin{array}{c}4 k-1 \\ 2\end{array}\right)+\frac{3}{2}(n-4 k+1),\left\lfloor\frac{2(k-1)(2 k-1)+(4 k-1)(n-2 k+1)}{2}\right\rfloor\right\}$.

If the conjecture is true, then the bound on size is best possible, which can be seen as following examples in [7]: Let $G_{1}$ be $K_{1}+\left(K_{4 k-2} \cup \frac{n-4 k+1}{2} K_{2}\right)$. The order of $G_{1}$ is $n$ and size $\left(\begin{array}{c}4 k-1 \\ 2\end{array}\right)+\frac{3}{2}(n-4 k+1)$, but $G_{1}$ does not contain $k$ disjoint theta graphs. Also, let $n$ be an integer such that $n-(2 k-1)$ is even. Let $l_{1}=\frac{n-(2 k-1)}{2}, F=K_{2 k-1}, H_{1}=l_{1} K_{2}$ and $G_{2}=F+H_{1}$. It is obvious that the graph $G_{2}$ has order $n,\left|E\left(G_{1}\right)\right|=(k-1)(2 k-1)+(4 k-1) l_{1}=$ $(k-1)(2 k-1)+\frac{(4 k-1)(n-2 k+1)}{2}=\left\lfloor\frac{2(k-1)(2 k-1)+(4 k-1)(n-2 k+1)}{2}\right\rfloor$. Gao and Ji [7] verified Conjecture 1.6 for the case $k=2$.

Theorem $1.7([7])$. Every graph of order $n \geq 8$ and size at least $f(n)$ contains two disjoint theta graphs, if

$$
f(n)= \begin{cases}23 & \text { if } n=8 \\ \left\lfloor\frac{7 n-13}{2}\right\rfloor & \text { if } n \geq 9 .\end{cases}
$$

Based on Theorem 1.7, in this paper, we give a sufficient condition for the existence of three disjoint theta graphs.

Theorem 1.8. Every graph of order $n \geq 12$ and size at least $\left\lfloor\frac{11 n-18}{2}\right\rfloor$ contains three disjoint theta graphs.

Note that there is a small gap on the lower bound of size between Theorem 1.8 and Conjecture 1.6 for $k=3$. However, the following corollary follows from Theorem 1.8.

Corollary 1.9. Every graph of order $n \geq 12$ and size at least $\left\lfloor\frac{11 n-18}{2}\right\rfloor$ contains three disjoint cycles of even length.

\section{Basic lemma}

Lemma 2.1. Let $G$ be a graph of order 12 and size at least $5 \%$. Then $G$ contains three disjoint copies of $K_{4}^{-}$.

Proof. Suppose that $G$ does not contain three disjoint copies of $K_{4}^{-}$. If $\delta(G) \geq$ 8, then by Theorem 1.5, $G \supseteq 3 K_{4}^{-}$, a contradiction. Hence, we may assume that $\delta(G) \leq 7$. Let $v_{0} \in V(G)$ such that $d_{G}\left(v_{0}\right)=\delta(G)$. Suppose that $d_{G}\left(v_{0}\right)=1$, then $56=|E(G)|<57$, a contradiction. Thus, $d_{G}\left(v_{0}\right) \geq 2$ and let $v_{1}, v_{2} \in N_{G}\left(v_{0}\right)$. Suppose that $d_{G}\left(v_{0}\right)=2$, then choose $w \in V(G-$ $\left.\left\{v_{0}, v_{1}, v_{2}\right\}\right)$, since $\left|E\left(G-\left\{v_{0}\right\}\right)\right| \geq 55$, it is obvious that $\left\{v_{0}, v_{1}, v_{2}, w\right\} \supseteq K_{4}^{-}$ and $\left[V(G)-\left\{v_{0}, v_{1}, v_{2}, w\right\}\right] \supseteq 2 K_{4}^{-}$, a contradiction. Hence, we may assume that $d_{G}\left(v_{0}\right) \geq 3$. Furthermore, since $G-\left\{v_{0}\right\}$ can be obtained from $K_{11}$ by removing at most five edges, it follows that $\left[N_{G}\left(v_{0}\right)\right]$ contains a path of order three, denoted by $P_{3}$. That is, $P_{3}+\left\{v_{0}\right\}$ contains a subgraph $Q \cong K_{4}^{-}$. Note 
that $\mid E\left(G-V(Q)-\left\{v_{0}\right\} \mid \geq 57-7-(10+9+8)=23\right.$, by Theorem 1.7, $G-V(Q)-\left\{v_{0}\right\}$ contains two disjoint copies of $K_{4}^{-}$, which disjoints from $Q$, this implies that $G \supseteq 3 K_{4}^{-}$, a contradiction. This proves Lemma 2.1.

\section{Proof of Theorem 1.8}

If $n=12$, then Lemma 2.1 gives us the required conclusion. Hence, it is sufficient to prove that every graph of order $n \geq 13$ and size at least $\left\lfloor\frac{11 n-18}{2}\right\rfloor$ contains three disjoint theta graph. We employ induction on $n$.

Assume that for all integers $k$ with $12 \leq k<n$, every graph of order $k$ and size at least $\left\lfloor\frac{11 k-18}{2}\right\rfloor$ contains three disjoint theta graphs. In the following proof, we always let $G$ be any graph of order $n$ and size at least $\left\lfloor\frac{11 n-18}{2}\right\rfloor$. By way of contradiction, we suppose that

$$
G \text { does not contain three disjoint theta graphs. }
$$

Claim 3.1. $6 \leq \delta(G) \leq 8$.

Proof. By Theorem 1.3, we have $\delta(G) \leq 8$. Suppose that $\delta(G) \leq 5$ and let $v_{0} \in V(G)$ such that $d_{G}\left(v_{0}\right)=\delta(G)$. The graph $G-v_{0}$ is of order $n-1$ and size $\left\lfloor\frac{11 n-18}{2}\right\rfloor-d_{G}\left(v_{0}\right) \geq\left\lfloor\frac{11 n-18}{2}\right\rfloor-5 \geq \frac{11 n-19-10}{2}=\frac{11(n-1)-18}{2} \geq\left\lfloor\frac{11(n-1)-18}{2}\right\rfloor$, by induction hypothesis, $G-v_{0}$ contains three disjoint theta graphs, and so does $G$, which contradicts (1). Therefore, $\delta(G) \geq 6$.

Let $v_{0}$ be a vertex in $G$ such that $d_{G}\left(v_{0}\right)=\delta(G)$. In what following, we always assume that $N_{G}\left(v_{0}\right)=\left\{v_{1}, \ldots, v_{l}\right\}$ and $H=\left[v_{1}, \ldots, v_{l}\right]$, where $l=$ $d_{G}\left(v_{0}\right)$. By Claim 3.1, $6 \leq l \leq 8$. If $l=6$, then let $\varepsilon_{l}=1$; if $l=7$, then let $\varepsilon_{l}=2$; if $l=8$, then let $\varepsilon_{l}=3$. Note that $l=5+\varepsilon_{l}$.

Claim 3.2. For each $1 \leq i \leq l, d_{H}\left(v_{i}\right) \geq l-\varepsilon_{l}$.

Proof. Suppose that there exists $1 \leq i \leq l$ such that $d_{H}\left(v_{i}\right) \leq l-\varepsilon_{l}-1=$ $(l-1)-\varepsilon_{l}$. Without loss of generality, we may assume that $i=l$, and we may also assume that $v_{j} v_{l} \notin E(G)$ for each $1 \leq j \leq \varepsilon_{l}$ (otherwise, we can relabel the index of $V(H))$. Define the edge set $X=\left\{v_{j} v_{l}: 1 \leq j \leq \varepsilon_{l}\right\}$ and construct the graph $G^{\prime}=\left(G-v_{0}\right)+X$, which is a graph with order $n-1$ and $\left|E\left(G^{\prime}\right)\right|=\left\lfloor\frac{11 n-18}{2}\right\rfloor-l+\varepsilon_{l} \geq \frac{11 n-19}{2}-l+\varepsilon_{l}=\frac{11(n-1)-18}{2} \geq\left\lfloor\frac{11(n-1)-18}{2}\right\rfloor$, because of $l=5+\varepsilon_{l}$. By induction hypothesis, $G^{\prime}$ contains three disjoint theta graphs, say $T_{1}, T_{2}$ and $T_{3}$, respectively. Clearly, at least two of them, say $T_{1}$ and $T_{2}$, do not contain vertex $v_{l}$, since $T_{1}, T_{2}$ and $T_{3}$ are disjoint theta graphs, then $E\left(T_{1}\right) \cap X=\emptyset, E\left(T_{2}\right) \cap X=\emptyset$ and by $(1), E\left(T_{3}\right) \cap X \neq \emptyset$.

Suppose that $\left|E\left(T_{3}\right) \cap X\right|=1$, we may assume that $E\left(T_{3}\right) \cap X=\left\{v_{l} v_{1}\right\}$. Then $T_{3}{ }^{\prime}=\left(T_{3}-\left\{v_{l} v_{1}\right\}\right)+\left\{v_{1} v_{0}, v_{l} v_{0}\right\}$ is a theta graph in $G, T_{1}, T_{2}$ and $T_{3}{ }^{\prime}$ are disjoint in $G$, which contradicts (1). Therefore, it remains the case 
$E\left(T_{3}\right) \cap X=\left\{v_{1} v_{l}, v_{2} v_{l}\right\}$ or $E\left(T_{3}\right) \cap X=\left\{v_{1} v_{l}, v_{2} v_{l}, v_{3} v_{l}\right\}$, as $\varepsilon_{l} \leq 3$. Let $T_{3}^{\prime}= \begin{cases}\left(T_{3}-\left\{v_{1} v_{l}, v_{2} v_{l}\right\}\right)+\left\{v_{0} v_{1}, v_{0} v_{2}\right\}, & \text { if } d_{T_{3}}\left(v_{l}\right)=2 \\ \left(T_{3}-\left\{v_{1} v_{l}, v_{2} v_{l}\right\}\right)+\left\{v_{0} v_{1}, v_{0} v_{2}, v_{0} v_{3}\right\}, & \text { if } d_{T_{3}}\left(v_{l}\right)=3 \text { and } \\ \left(T_{3}-\left\{v_{1} v_{l}, v_{2} v_{l}, v_{3} v_{l}\right\}\right)+\left\{v_{0} v_{1}, v_{0} v_{2}, v_{0} v_{3}\right\}, & E\left(T_{3}\right) \cap X=\left\{v_{1} v_{l}, v_{2} v_{l}\right\} \\ \text { otherwise. }\end{cases}$

It is obvious that $T_{1}, T_{2}$ and $T_{3}{ }^{\prime}$ are three disjoint theta graphs in $G$, which contradicts (1).

By Claim 3.2, Theorem 1.5 and the definition of $\varepsilon_{l}$, when $7 \leq l \leq 8$, for each subset $S$ of $V(H)$ with $|S| \geq 7$, we obtain

$$
\left[\left\{v_{0}\right\} \cup S\right] \supseteq 2 K_{4}^{-} \text {. }
$$

In particular, if $l=6$, then

$$
\left[\left\{v_{0}\right\} \cup V(H)\right] \cong K_{7} .
$$

We take a vertex $v \in V\left(G-H-\left\{v_{0}\right\}\right)$ such that $|E(v, V(H))|$ is maximum. When $l=6$, by (3) and the definition of $v$, denote $W=V(H) \cup\{v\}$, we claim that

$$
\left[\left\{v_{0}\right\} \cup W\right] \supseteq 2 K_{4}^{-} .
$$

Proof. By way of contradiction, suppose that $\left[\left\{v_{0}\right\} \cup W\right]$ does not contain two disjoint $K_{4}^{-}$. By $(3)$ and the assumption that $\left[\left\{v_{0}\right\} \cup W\right] \nsupseteq 2 K_{4}^{-}$, for each $w \in V\left(G-\left\{v_{0}\right\}-V(H)\right)$, there is at most one edge between $w$ and $V(H)$. If $n=13$, then $62 \leq|E(G)| \leq \frac{7 \times 6}{2}+6+\frac{6 \times 5}{2}=42$, a contradiction. If $n=14$, then $68 \leq|E(G)| \leq \frac{7 \times 6}{2}+7+\frac{7 \times 6}{2}=49$, a contradiction. If $n=15$, then $73 \leq|E(G)| \leq \frac{7 \times 6}{2}+8+\frac{8 \times 7}{2}=57$, a contradiction. If $n=16$, then $84 \leq|E(G)| \leq \frac{7 \times 6}{2}+9+\frac{9 \times 8}{2}=66$, a contradiction. Therefore, we see that $n \geq 17$. Since

$$
\begin{aligned}
\left|E\left(G-\left\{v_{0}\right\}-V(H)\right)\right| & \geq|E(G)|-\frac{7 \times 6}{2}-(n-7) \\
& \geq \frac{11 n-19}{2}-n-14 \\
& \geq \frac{7 n-13}{2}
\end{aligned}
$$

by Theorem 1.7, $G-\left\{v_{0}\right\}-V(H)$ contains two disjoint theta graphs, together with (3), $G$ contains three disjoint theta graphs, a contradiction.

Let

$$
G^{*}= \begin{cases}G-\left(\left\{v_{0}\right\} \cup V(H)\right), & \text { if } 7 \leq l \leq 8 \\ G-\left(\left\{v_{0}, v\right\} \cup V(H)\right), & \text { if } l=6 .\end{cases}
$$

Let $F^{*}$ be the set of components of $G^{*}$. By (2) and (4), it follows from (1) that every graph in $F^{*}$ contains no theta graph. In the following proof, let $F$ denote arbitrary component in $F^{*}$, then, each block of $F$ is either a $K_{2}$ or a cycle. 
Claim 3.3. Let $F \in F^{*}$ with $|V(F)| \geq 4$. Then each end block of $F$ is isomorphic to $\mathrm{K}_{2}$.

Proof. Otherwise, suppose that there exists an end block $B$ of $F$, such that $B$ is a cycle. Let $C$ denote the set of cut vertices of $F$. Let $u_{1}$ and $u_{2}$ be two distinct vertices in $V(B)-C$. Next, we choose two distinct vertices $u_{3}$ and $u_{4}$ (both are distinct with $u_{1}$ and $u_{2}$ ) as follows: If $F=B$, then let $\left\{u_{3}, u_{4}\right\} \subseteq V\left(F-\left\{u_{1}, u_{2}\right\}\right)$; otherwise, $F$ contains another end blocks $B^{\prime}$ which is different from $B$, let $u_{3} \in V\left(B^{\prime}\right)$ such that $u_{3} \notin C$ and choose $u_{4} \in V(F) \backslash C$ if possible, unless $F$ contains exactly two end blocks $B$ and $B^{\prime}$, such that $B$ is a triangle and $B^{\prime} \cong K_{2}$. For each $i$ with $1 \leq i \leq 3$, since $d_{F}\left(u_{i}\right) \leq 2$, if $7 \leq l \leq 8$, then $\left|E\left(u_{i}, V(H)\right)\right| \geq \delta(G)-2=l-2$, if $\bar{l}=6$, then $\left|E\left(u_{i}, V(H) \cup\{v\}\right)\right| \geq l-2$. This implies that there exists a vertex $v^{\prime} \in V(H)\left(v^{\prime} \in V(H) \cup\{v\}\right.$ if $\left.l=6\right)$, such that $u_{1} v^{\prime}, u_{2} v^{\prime} \in E(G)$. As $B$ is a cycle, it is easy to see that $\left[B \cup\left\{v^{\prime}\right\}\right]$ contains a theta graph. When $F=B$, without loss of generality, we may assume that $u_{1}, u_{2}, u_{3}$ and $u_{4}$ occur along the direction of $B$.

If $l=8$, by applying (2) and Theorem 1.5, $\left[\left\{v_{0}\right\} \cup V(H)-\left\{v^{\prime}\right\}\right]$ contains two theta graphs, that is, $G$ contains three disjoint theta graphs, which contradicts (1). If $l=7$, we may assume that $\left\{v_{2}, v_{3}, v_{4}, v_{5}, v_{6}\right\} \subseteq N_{G}\left(u_{3}\right)$ and $v^{\prime} \neq v_{4}, v_{5}$ and $v_{6}$, then $\left[\left\{v_{4}, v_{5}, v_{6}, u_{3}\right\}\right] \supseteq K_{4}^{-}$by Claim 3.2. If $u_{3} \notin V(B)$, that is, $u_{3}$ belongs to another end block by our choice, notice that $\left[V\left(H-\left\{v_{4}, v_{5}, v_{6}, v^{\prime}\right\}\right) \cup\right.$ $\left.\left\{v_{0}\right\}\right] \supseteq K_{4}^{-}$and $\left[B \cup\left\{v^{\prime}\right\}\right]$ contains a theta graph, we obtain a contradiction to (1). Therefore, we see that $u_{3} \in V(B)$ and so $F=B$ by our choice. We may assume that $\left\{v_{1}, v_{2}, v_{3}\right\} \subseteq N_{G}\left(u_{1}\right) \cap N_{G}\left(u_{2}\right)$ because we don't use the assumption of $\left\{v_{2}, v_{3}, v_{4}, v_{5}, v_{6}\right\} \subseteq N_{G}\left(u_{3}\right)$. Suppose for the moment, there exists at most one $v_{i} \in\left\{v_{1}, v_{2}, v_{3}\right\}$, such that $v_{i} u_{3}, v_{i} u_{4} \in E(G)$. Then there exist $v_{p}, v_{q} \in V\left(H-\left\{v_{1}, v_{2}, v_{3}\right\}\right)$ with $p \neq q$, such that $\left\{v_{p}, v_{q}\right\} \subseteq N_{G}\left(u_{3}\right) \cap N_{G}\left(u_{4}\right)$. However, by Claim 3.2, $\left[\left\{v_{0}\right\} \cup V\left(H-\left\{v_{1}, v_{2}, v_{p}, v_{q}\right\}\right)\right] \supseteq K_{4}^{-}$, notice that $\left[\left\{v_{1}, v_{2}\right\} \cup V\left(\vec{B}\left[u_{1}, u_{2}\right]\right)\right]$ and $\left[\left\{v_{p}, v_{q}\right\} \cup V\left(\vec{B}\left[u_{3}, u_{4}\right]\right)\right]$ contain two disjoint theta graphs, this implies that $G$ contains three disjoint theta graphs, a contradiction. Thus, without loss of generality, say $\left\{v_{1}, v_{2}\right\} \subseteq N_{H}\left(u_{3}\right) \cap N_{H}\left(u_{4}\right)$. As $\left|E\left(u_{3}, V(H)\right)\right| \geq 5$, without loss of generality, we may assume that $v_{4} u_{3}, v_{5} u_{3} \in$ $E(G)$. As $d_{H}\left(v_{1}\right) \geq 5$ by Claim 3.2 , we may assume that $v_{1} v_{4} \in E(G)$. This implies that $\left[v_{4}, v_{1}, u_{4}, u_{3}\right] \supseteq K_{4}^{-}$, notice that $\left[\left\{v_{0}, v_{5}, v_{6}, v_{7}\right\}\right] \supseteq K_{4}^{-}$and $\left[\left\{v_{2}, v_{3}\right\} \cup V\left(\vec{B}\left[u_{1}, u_{2}\right]\right)\right]$ contains a theta graph, then $G$ contains three disjoint theta graphs, a contradiction. Now, it remains the case $l=6$. As $d_{F}\left(u_{i}\right) \leq 2$ for $i \in\{1,2,3\}$, so $\left|E\left(u_{i}, V(H) \cup\{v\}\right)\right| \geq l-2=4$. Furthermore, by our choice of $u_{4}, d_{F}\left(u_{i}\right) \leq 3$ and $\left|E\left(u_{4}, V(H) \cup\{v\}\right)\right| \geq 3$.

Suppose for the moment that $u_{1} v, u_{2} v, u_{3} v \in E(G)$, then $[B \cup\{v\}]$ contains a theta graph. If $u_{3} \notin V(B)$, by the choice of $u_{3}$ and (3), $H+\left\{v_{0}, u_{3}\right\} \supseteq 2 K_{4}^{-}$, this implies that $G$ contains three disjoint theta graphs, which contradicts (1). Thus, $u_{3} \in V(B)$ and so $F=B$. However, $\left[\{v\} \cup V\left(\vec{B}\left[u_{1}, u_{3}\right]\right)\right]$ contains a theta graph and $\left[\left\{u_{4}, v_{0}\right\} \cup V(H)\right] \supseteq 2 K_{4}^{-}$, a contradiction. Thus, there exists 
$i \in\{1,2,3\}$, such that $u_{i} v \notin E(G)$. By the definition of $v,|E(v, V(H))| \geq 4$. Without loss of generality, we assume that $\left\{v_{1}, v_{2}, v_{3}, v_{4}\right\} \subseteq N_{H}(v)$.

If $u_{1} v, u_{2} v \in E(G)$, then $F=B$. Without loss of generality, we may assume that $v_{4} u_{1} \in E(G)$ and so $\left[\left\{v, v_{4}\right\} \cup V\left(\vec{B}\left[u_{1}, u_{2}\right]\right)\right]$ contains a theta graph. Notice that $u_{3} v \notin E(G)$ and $u_{4} v \notin E(G)$, then $\left[\left\{u_{3}, u_{4}, v_{0}\right\} \cup V\left(H-\left\{v_{4}\right\}\right)\right] \supseteq 2 K_{4}^{-}$, a contradiction. Therefore, we assume that $u_{1} v \notin E(G)$ by symmetry.

Suppose that $u_{3} \notin V(B)$. Then $\left|E\left(u_{3},\left\{v_{1}, v_{2}, v_{3}, v_{4}\right\}\right)\right| \geq 2$; otherwise, $u_{3} v \in E(G)$ and we may further assume that $u_{3} v_{4}, u_{3} v_{5}, u_{3} v_{6} \in E(G)$. Since $\left|E\left(u_{1}, V(H)\right)\right| \geq 4$ and $\left|E\left(u_{2}, V(H)\right)\right| \geq 3$, there exists $i \in\{1,2,3,4\}$, such that $u_{i} \in N_{H}\left(u_{2}\right) \cap N_{H}\left(u_{1}\right)$, then $\left[B \cup\left\{u_{i}\right\}\right]$ is a theta graph, since $\left[\left\{u_{1}, u_{2}, u_{3}, u_{4}, v\right\}-\right.$ $\left.\left\{u_{i}\right\}\right] \supseteq K_{4}^{-}$and $\left[v_{0}, v_{5}, v_{6}, u_{3}\right] \supseteq K_{4}^{-}$, which contradicts (1). By symmetry, we assume that $v_{1} u_{3}, v_{2} u_{3} \in E(G)$. If $v^{\prime} \neq v_{1}$ and $v^{\prime} \neq v_{2}$, then $\left[v, u_{3}, v_{1}, v_{2}\right] \supseteq K_{4}^{-}$ and $\left[\left\{v_{0}\right\} \cup\left(V(H)-\left\{v_{1}, v_{2}, v^{\prime}\right\}\right)\right] \supseteq K_{4}^{-}$, which disjoints from $\left[B \cup\left\{v^{\prime}\right\}\right]$, a contradiction. Hence, we may assume that $v^{\prime}=v_{1}$. By Claim 3.2 and (1), we may assume that $v_{5} u_{3}, v_{6} u_{3} \in E(G)$. However, $\left[v_{0}, v_{5}, v_{6}, u_{3}\right] \supseteq K_{4}^{-}$and $\left[v, v_{2}, v_{3}, v_{4}\right] \supseteq K_{4}^{-}$, which disjoints from $\left[B \cup\left\{v_{1}\right\}\right]$, a contradiction. Thus, $u_{3} \in V(B)$ and so $F=B$ by our choice.

By symmetry, we may assume that $u_{2} v \notin E(G)$ and $u_{3} v \notin E(G)$. By pigeonhole principle, there exists $\left\{v_{p}, v_{q}\right\} \subseteq V(H)$ such that $\left\{v_{p}, v_{q}\right\} \subseteq N_{H}\left(u_{1}\right) \cap$ $N_{H}\left(u_{2}\right)$. If $u_{3} v_{p} \in E(G)$, then $\left[V\left(\vec{B}\left[u_{1}, u_{3}\right]\right) \cup\left\{v_{p}\right\}\right]$ contains a theta graph, notice that $\left[V\left(H-\left\{v_{p}\right\}\right) \cup\left\{v, v_{0}, u_{4}\right\}\right] \supseteq 2 K_{4}^{-}, G$ contains three disjoint theta graphs, a contradiction. Thus, $u_{3} v_{p} \notin E(G)$ and by symmetry, $u_{3} v_{q} \notin$ $E(G), u_{4} v_{p} \notin E(G)$ and $u_{4} v_{q} \notin E(G)$. This implies that there exist $v_{i}, v_{j} \in$ $V(H)-\left\{v_{p}, v_{q}\right\}$, such that $\left\{v_{i}, v_{j}\right\} \subseteq N_{H}\left(u_{3}\right) \cap N_{H}\left(u_{4}\right)$. By (1), we see that $|\{p, q\} \cap\{1,2,3,4\}| \leq 1$ and $|\{i, j\} \cap\{1,2,3,4\}| \leq 1$. Therefore, $\left[\left\{v, v_{0}\right\} \cup V(H-\right.$ $\left.\left.\left\{v_{p}, v_{q}, v_{i}, v_{j}\right\}\right)\right] \supseteq K_{4}^{-}$. Notice that $\left[\left\{v_{p}, v_{q}\right\} \cup V\left(\vec{B}\left[u_{1}, u_{2}\right]\right)\right]$ and $\left[\left\{v_{i}, v_{j}\right\} \cup\right.$ $\left.V\left(\vec{B}\left[u_{3}, u_{4}\right]\right)\right]$ contains two disjoint theta graphs, which contradicts (1). This completes the proof that $B$ is not an end block, and in particular, we see that every end block of $F$ is isomorphic $K_{2}$.

Claim 3.4. Let $F \in F^{*}$ with $|V(F)| \geq 4$. Then each block of $F$ is isomorphic to $K_{2}$.

Proof. Since $|V(F)| \geq 4, F$ contains at least two end block, say $F_{1}$ and $F_{2}$. Note $F_{i} \cong K_{2}$ for each $1 \leq i \leq 2$. Let $u_{1} \in V\left(F_{1}\right)$ such that $d_{F_{1}}\left(u_{1}\right)=1$ and let $u_{3} \in V\left(F_{2}\right)$ such that $d_{F_{2}}\left(u_{3}\right)=1$. Suppose that the conclusion of Claim 3.4 is false, we may assume that $B$ is the nearest block to $u_{1}$ in $F$, such that $B$ is a cycle. By Claim 3.3, $B$ is not an end block of $F$. We choose two distinct vertices $u_{2}$ and $u_{4}$ such that both of them are distinct with $u_{1}$ and $u_{3}$ as follows: Let $u_{2} \in V(B)$ and $u_{2}$ is not a cut vertex of $F$, and choose $u_{4}$ such that $u_{4}$ is not a cut vertex of $F$, unless $F$ contains exactly three blocks $F_{1}, F_{2}$ and $B \cong K_{3}$, then choose $u_{4} \in V\left(F_{2}\right)-\left\{u_{3}\right\}$. Notice that if there exists $v^{\prime}$ such that $u_{1} v^{\prime}, u_{2} v^{\prime} \in E(G)$, then using these blocks of $F$ from $F_{1}$ to $B$, we see that $\left[V\left(F-\left\{u_{3}\right\}\right) \cup\left\{u_{1}\right\}\right]$ contains a theta graph. Now, since $u_{1}$ and $u_{3}$ are 
in different blocks, with the same role of $u_{1}, u_{2}, u_{3}$ and $u_{4}$, we continue part of the process in the proof of Claim 3.3, we can complete the proof. This proves Claim 3.4.

Claim 3.5. $|V(F)| \leq 3$ for each $F \in F^{*}$.

Proof. Otherwise, suppose that there exists $F \in F^{*}$ such that $|V(F)| \geq 4$. By Claim 3.4, $F$ must be a tree.

Suppose for the moment that there exists three distinct leaves in $V(F)$, say $u_{1}, u_{2}$ and $u_{3}$. Then for each $1 \leq i \leq 3,\left|E\left(u_{i}, V(H)\right)\right| \geq l-1$ if $7 \leq l \leq 8$, and $\left|E\left(u_{i}, V(H) \cup\{v\}\right)\right| \geq l-1$ if $l=6$. As $|V(F)| \geq 4$, by Claim 3.4, we choose $u_{4} \in V\left(F-\left\{u_{1}, u_{2}, u_{3}\right\}\right)$ as follows: if $F$ contains at least four leaves, then let $u_{4}$ denote the leave different from $u_{1}, u_{2}$ and $u_{3}$; otherwise, let $u_{4}$ and $u_{1}$ belongs to the same block of $F$. It is obvious that $\left|E\left(u_{4}, V(H)\right)\right| \geq l-3$ if $7 \leq l \leq 8$, and $\left|E\left(u_{4}, V(H) \cup\{v\}\right)\right| \geq l-3$ if $l=6$.

Suppose that $l=8$. Notice that there exist $v^{\prime}, v^{\prime \prime} \in V(H)$ with $v^{\prime} \neq v^{\prime \prime}$ and $v^{\prime} v^{\prime \prime} \in E(G)$ such that $\left\{v^{\prime}, v^{\prime \prime}\right\} \subseteq N_{H}\left(u_{1}\right) \cap N_{H}\left(u_{2}\right)$. It is obvious that $\left[v^{\prime}, v^{\prime \prime}, u_{1}, u_{2}\right] \supseteq K_{4}^{-}$. by Claim 3.1, $H-\left\{v^{\prime}, v^{\prime \prime}\right\}+\left\{v_{0}, u_{3}\right\}$ induces a graph with minimum degree at least five, and therefore contains two disjoint copies of $K_{4}^{-}$by Theorem 1.5 , a contradiction. Next, suppose that $l=7$, by pigeonhole principle, we can find two distinct vertices $v_{i}, v_{j} \in V(H)$ such that $\left\{v_{i}, v_{j}\right\} \subseteq$ $N_{H}\left(u_{3}\right) \cap N_{H}\left(u_{4}\right)$. Since there is a path $P$ in $F$ which connecting $u_{3}$ and $u_{4}$, thus, $\left[V(P) \cup\left\{v_{i}, v_{j}\right\}\right]$ contains a theta graph. Notice that there exist $v^{\prime}, v^{\prime \prime} \in V\left(H-\left\{v_{i}, v_{j}\right\}\right)$ with $v^{\prime} \neq v^{\prime \prime}$ and $v^{\prime} v^{\prime \prime} \in E(G)$, such that $\left\{v^{\prime}, v^{\prime \prime}\right\} \subseteq$ $N_{H}\left(u_{1}\right) \cap N_{H}\left(u_{2}\right)$. It is obvious that $\left[v^{\prime}, v^{\prime \prime}, u_{1}, u_{2}\right] \supseteq K_{4}^{-}$. As $\left[\left\{v_{0}\right\} \cup V(H-\right.$ $\left.\left.\left\{v^{\prime}, v^{\prime \prime}, v_{i}, v_{j}\right\}\right)\right] \supseteq K_{4}^{-}$, which contradicts (1). Thus, $l=6$.

We show $N_{H}\left(u_{1}\right) \cap N_{H}\left(u_{4}\right) \neq \emptyset$. Suppose not, without loss of generality, we may assume that $N_{G}\left(u_{1}\right) \cap(V(H) \cup\{v\})=\left\{v, v_{1}, v_{2}, v_{3}, v_{4}\right\}$ and $N_{G}\left(u_{4}\right) \cap$ $(V(H) \cup\{v\})=\left\{v, v_{5}, v_{6}\right\}$. If $u_{3} v \in E(G)$, then $\left[V\left(F-\left\{u_{2}\right\}\right) \cup\{v\}\right]$ contains a theta graph, as $\left[V(H) \cup\left\{v_{0}, u_{2}\right\}\right] \supseteq 2 K_{4}^{-}$, which contradicts (1). Hence, $u_{3} v \notin E(G)$ and $u_{2} v \notin E(G)$ by symmetry. Furthermore, by the choice of $v$, we have $|E(v, V(H))| \geq 4$ and so $N_{H}(v) \cap N_{H}\left(u_{1}\right) \neq \emptyset$, without loss of generality, say $v v_{1} \in E(G)$. Then $\left[v, v_{1}, u_{1}, u_{4}\right] \supseteq K_{4}^{-}$, since $\left|N_{H}\left(u_{2}\right) \cap N_{H}\left(u_{3}\right)\right| \geq 3$, it follows that $\left[V\left(H-\left\{v_{1}\right\}\right) \cup\left\{u_{2}, u_{3}, v_{0}\right\}\right] \supseteq 2 K_{4}^{-}$, which contradicts (1).

Now, by symmetry, say $v_{6} \in N_{H}\left(u_{1}\right) \cap N_{H}\left(u_{4}\right)$. If $u_{2} v_{6} \in E(G)$, then $\left[\left\{v_{6}\right\} \cup V\left(F-\left\{u_{3}\right\}\right)\right]$ contains a theta graph, as $\left[V\left(H-\left\{v_{6}\right\}\right) \cup\left\{v, u_{3}\right\}\right] \supseteq 2 K_{4}^{-}$, which contradicts (1). Thus, $v_{6} u_{2} \notin E(G)$ and $v_{6} u_{3} \notin E(G)$ by symmetry. As $\left|E\left(u_{2}, V(H)\right)\right| \geq 4$ and $\left|E\left(u_{3}, V(H)\right)\right| \geq 4$, we may assume that $\left\{v_{1}, v_{2}, v_{3}, v_{4}\right\} \subseteq N_{H}\left(u_{2}\right)$ and $\left\{v_{1}, v_{2}, v_{3}\right\} \subseteq N_{H}\left(u_{2}\right) \cap N_{H}\left(u_{3}\right)$. If $v_{5} u_{1} \in E(G)$, then $\left[\left\{v_{6}, v_{5}, u_{1}, u_{4}\right\}\right] \supseteq K_{4}^{-}$. Notice that $\left[V\left(H-\left\{v_{5}, v_{6}\right\}\right) \cup\left\{v_{0}, v, u_{3}, u_{2}\right\}\right] \supseteq$ $2 K_{4}^{-}$by the definition of $v$ and (3), which contradicts (1). Thus, $v_{5} u_{1} \notin E(G)$. If $u_{1} v_{4} \in E(G)$, then $u_{2} v, u_{3} v \in E(G)$. Otherwise, say $u_{2} v \notin E(G)$. Then $u_{2} v_{5} \in E(G)$ and $|E(v, V(H))| \geq 5$ by the choice of $v$. By symmetry, we may assume that $\left\{v_{1}, v_{2}\right\} \subseteq N_{H}(v) \cap N_{H}\left(u_{3}\right)$. Then $\left[v, v_{1}, v_{2}, u_{3}\right] \supseteq K_{4}^{-}$, $\left[u_{1}, u_{4}, v_{4}, v_{6}\right] \supseteq K_{4}^{-}$and $\left[u_{2}, v_{3}, v_{5}, v_{0}\right] \supseteq K_{4}^{-}$, which contradicts (1). Hence, 
by (1), $v v_{i} \notin E(G)$ for each $i \in\{1,2,3\}$, that is, $|E(v, V(H))| \leq 3$, which contradicts the choice of $v$. Therefore, $u_{1} v_{4} \notin E(G)$ and so $\left\{v_{1}, v_{2}, v_{3}\right\} \subseteq N_{H}\left(u_{1}\right)$ and $u_{1} v \in E(G)$. By (1) and (3), $u_{2} v, u_{3} v \in E(G)$ and $|E(v, V(H))| \leq 3$, which contradicts the choice of $v$. Consequently, $F$ contains exactly two leaves and $F$ must be a path with order at least four.

Let $F=u_{1} u_{2} \cdots u_{p-1} u_{p}$ and $p \geq 4$. Suppose that $7 \leq l \leq 8$, then continue the process as above, we can find three disjoint theta graphs, a contradiction. Hence, $l=6$. Then $\left|E\left(u_{1}, V(H) \cup\{v\}\right)\right| \geq 5,\left|E\left(u_{p}, V(H) \cup\{v\}\right)\right| \geq 5$, $\left|E\left(u_{2}, V(H) \cup\{v\}\right)\right| \geq 4$ and $\left|E\left(u_{p-1}, V(H) \cup\{v\}\right)\right| \geq 4$.

Suppose $u_{1} v, u_{p} v \in E(G)$. Then $u_{2} v \notin E(G)$ or $u_{p-1} v \notin E(G)$, otherwise, $\left[v, u_{1}, u_{2}, u_{p-1}\right] \supseteq K_{4}^{-}$, as $\left[V(H) \cup\left\{v_{0}, u_{p}\right\}\right] \supseteq 2 K_{4}^{-}$by Claim 3.2, which contradicts (1). By symmetry, say $u_{2} v \notin E(G)$ and so $\left|E\left(u_{2}, V(H)\right)\right| \geq 4$. Without loss of generality, by pigeonhole principle, we may assume that $v_{1} \in$ $N_{H}\left(u_{2}\right) \cap N_{H}\left(u_{p-1}\right)$ and $\left\{v_{1}, v_{2}, v_{3}, v_{4}\right\} \subseteq N_{H}\left(u_{2}\right)$. Suppose for a moment that $\left|N_{H}\left(u_{2}\right) \cap N_{H}\left(u_{p-1}\right)\right| \geq 2$. Without loss of generality, say $v_{2} u_{p-1} \in$ $E(G)$. Then $\left[u_{2}, u_{p-1}, v_{1}, v_{2}\right] \supseteq K_{4}^{-}$. We prove that $v v_{1} \notin E(G)$ and $v v_{2} \notin$ $E(G)$. Otherwise, by symmetry, say $v v_{1} \in E(G)$. If $u_{1} v_{1} \in E(G)$, then $\left[v, v_{1}, u_{1}, u_{2}\right] \supseteq K_{4}^{-}$, since $\left[\left\{u_{p}, v_{0}\right\} \cup V\left(H-\left\{v_{1}\right\}\right)\right] \supseteq 2 K_{4}^{-}$, which contradicts (1). Hence, $u_{1} v_{1} \notin E(G)$. Next, we show that $u_{1} v_{2} \notin E(G)$. Suppose that $u_{1} v_{2} \in E(G)$. Then $\left[V\left(F-\left\{u_{p}\right\}\right) \cup\left\{v_{2}\right\}\right]$ contains a theta graph, as $\left[\left\{v, u_{p}, v_{0}\right\} \cup V\left(H-\left\{v_{2}\right\}\right)\right] \supseteq 2 K_{4}^{-}$, a contradiction once again. Until now, we see that $N_{H}\left(u_{1}\right)=\left\{v_{3}, v_{4}, v_{5}, v_{6}\right\}$. According to this, we have $u_{p} v_{1} \notin E(G)$ and $u_{p} v_{2} \notin E(G)$. This implies that $N_{H}\left(u_{1}\right)=N_{H}\left(u_{p}\right)$. If $v v_{2} \in E(G)$, then $\left[v, v_{1}, v_{2}, u_{p-1}\right] \supseteq K_{4}^{-}$, notice that $\left[V\left(H-\left\{v_{1}, v_{2}\right\}\right) \cup\left\{u_{1}, u_{2}, u_{p}, v_{0}\right\}\right] \supseteq 2 K_{4}^{-}$, which contradicts (1). Thus, $v v_{2} \notin E(G)$ and it follows that there exists $i \in\{3,4\}$ such that $v_{i} v \in E(G)$. Without loss of generality, say $i=3$, then $\left[v, v_{3}, u_{1}, u_{2}\right] \supseteq K_{4}^{-}$, as $\left[V\left(H-\left\{v_{3}\right\}\right) \cup\left\{u_{p-1}, u_{p}, v_{0}\right\}\right] \supseteq 2 K_{4}^{-}$, which contradicts (1) and completes the proof of $v v_{1} \notin E(G)$. Then $\left\{v_{3}, v_{4}, v_{5}, v_{6}\right\} \subseteq N_{H}(v)$ and so $\left[V\left(H-\left\{v_{1}, v_{2}\right\}\right) \cup\left\{v, u_{p}, u_{1}, v_{0}\right\}\right] \supseteq 2 K_{4}^{-}$, a contradiction. This proves that $N_{H}\left(u_{2}\right) \cap N_{H}\left(u_{p-1}\right)=\left\{v_{1}\right\}$ and so $v_{5} u_{p-1}, v_{6} u_{p-1}, u_{p-1} v \in E(G)$. Suppose that $v_{1} u_{1} \in E(G)$, then let $P^{\prime}=P-\left\{u_{p}\right\}$, then $\left[V\left(P^{\prime}\right) \cup\left\{v_{1}\right\}\right]$ contains a theta graph, by $(3),\left[V\left(H-\left\{v_{1}\right\}\right) \cup\left\{v, u_{p}\right\}\right] \supseteq 2 K_{4}^{-}$, which contradicts (1). Thus, $v_{1} u_{1} \notin E(G)$ and so $\left|N_{H-v_{1}}\left(u_{1}\right) \cap N_{H-v_{1}}\left(u_{p}\right)\right| \geq 2$. If $v v_{1} \in E(G)$, then $\left[V\left(P-\left\{u_{1}, u_{p}\right\}\right) \cup\left\{v, v_{1}\right\}\right]$ contains a theta graph, as $\left[V\left(H-\left\{v_{1}\right\}\right) \cup\left\{u_{1}, u_{p}\right\}\right] \supseteq 2 K_{4}^{-}$, which contradicts (1). Thus, $v v_{1} \notin E(G)$. As $|E(v, V(H))| \geq 4$, by the symmetry role of $v_{5}$ and $v_{6}$, we may assume that $v v_{5} \in E(G)$, then $\left[v, v_{5}, u_{p-1}, u_{p}\right] \supseteq K_{4}^{-}$, since $u_{1}$ and $u_{2}$ has at least two common neighbors in $V(H)-\left\{v_{1}, v_{5}, v_{6}\right\},\left[V\left(H-\left\{v_{5}\right\}\right) \cup\left\{u_{1}, u_{2}\right\}\right] \supseteq 2 K_{4}^{-}$, which contradicts (1). Consequently, we may assume that $u_{1} v \notin E(G)$ by symmetry. This gives us $\left|E\left(u_{1}, V(H)\right)\right| \geq 5$ and so $|E(v, V(H))| \geq 5$ by the maximality of $v$. Without loss of generality, we may assume that $\left\{v_{1}, v_{2}, v_{3}, v_{4}, v_{5}\right\} \subseteq N_{H}(v)$ and $\left\{v_{1}, v_{2}, v_{3}, v_{4}\right\} \subseteq N_{H}\left(u_{1}\right) \cap N_{H}(v)$. Because of $\left|E\left(u_{1}, V(H)\right)\right| \geq 5$, we divide the proof into two cases. 
Case 1. $u_{1} v_{5} \in E(G)$.

Without loss of generality, say $v_{4} u_{2}, v_{5} u_{2} \in E(G)$, because of $\left|E\left(u_{2}, V(H)\right)\right|$ $\geq 3$. If $u_{p-1} v_{4} \in E(G)$, then $\left[u_{1}, u_{2}, \ldots, u_{p-1}, v_{4}\right]$ contains a theta graph, since $\left[V\left(H-\left\{v_{4}\right\}\right) \cup\left\{v, u_{p}, v_{0}\right\}\right] \supseteq 2 K_{4}^{-}$, which contradicts (1) and proves that $u_{p-1} v_{4} \notin E(G)$. Similarly, $u_{p-1} v_{5} \notin E(G)$. If there exists $v_{i} \in\left\{v_{1}, v_{2}, v_{3}\right\}$, say $i=1$, such that $v_{1} u_{2} \in E(G)$, then $u_{p-1} v_{1} \notin E(G), N_{H}\left(u_{p-1}\right)=\left\{v_{2}, v_{3}, v_{6}\right\}$ and $u_{p-1} v \in E(G)$. Suppose that there exist $v_{i}, v_{j} \in\left\{v_{1}, v_{4}, v_{5}\right\}$ such that $u_{p} v_{i}, u_{p} v_{j} \in E(G)$, then $\left[v_{i}, v_{j}, u_{2}, u_{p}\right] \supseteq K_{4}^{-}$. For simplicity, say $i=4$ and $j=5$. Since $\left[v, v_{2}, v_{6}, u_{p-1}\right] \supseteq K_{4}^{-}$and $\left[v_{0}, u_{1}, v_{1}, v_{3}\right] \supseteq K_{4}^{-}$, this contradicts (1) and proves that $u_{p}$ has at most one neighbor in $\left\{v_{1}, v_{4}, v_{5}\right\}$. This implies that $u_{p} v_{6}, u_{p} v \in E(G)$. Hence, $\left[v, u_{p-1}, u_{p}, v_{6}\right] \supseteq K_{4}^{-}$, notice that $\left[V\left(H-\left\{v_{6}\right\}\right) \cup\right.$ $\left.\left\{v_{0}, u_{1}, u_{2}\right\}\right] \supseteq 2 K_{4}^{-}$, a contradiction. This proves that $u_{2}$ has no neighbor in $\left\{v_{1}, v_{2}, v_{3}\right\}$ and so $u_{2} v_{6}, u_{2} v \in E(G)$. As $\left|E\left(u_{p-1}, V(H)\right)\right| \geq 3$, we may assume that $v_{2} u_{p-1}, v_{3} u_{p-1} \in E(G)$. Since $\left[v, v_{4}, u_{1}, u_{2}\right] \supseteq K_{4}^{-}$and $\left[v_{0}, v_{2}, v_{3}, u_{p-1}\right] \supseteq$ $K_{4}^{-},\left|E\left(u_{p},\left\{v_{1}, v_{5}, v_{6}\right\}\right)\right| \leq 1$ by (1) and (3). Therefore, $\left\{v_{2}, v_{3}, v_{4}\right\} \in N_{H}\left(u_{p}\right)$ and $u_{p} v \in E(G)$. However, $\left[v, v_{5}, v_{6}, u_{2}\right] \supseteq K_{4}^{-},\left[u_{p-1}, v_{2}, v_{3}, u_{p}\right] \supseteq K_{4}^{-}$and $\left[v_{0}, v_{1}, v_{4}, u_{1}\right] \supseteq K_{4}^{-}$, a contradiction. This proves Case 1 .

Case 2. $u_{1} v_{6} \in E(G)$.

Suppose that $u_{2} v \in E(G)$. Then for each $v_{i}$ with $1 \leq i \leq 4, v_{i} u_{2} \notin E(G)$, otherwise, $\left[v, v_{i}, u_{2}, u_{1}\right] \supseteq K_{4}^{-}$, it is obvious that $\left[V\left(H-\left\{v_{i}\right\}\right) \cup\left\{v_{0}, u_{p}, u_{p-1}\right\}\right] \supseteq$ $2 K_{4}^{-}$, which contradicts (1). However, this gives us $\left|E\left(u_{2}, V(H) \cup\{v\}\right)\right| \leq 3$, a contradiction. Thus, $u_{2} v \notin E(G)$ and $\left|E\left(u_{2}, V(H)\right)\right| \geq 4$. By symmetry, we may assume that $u_{2} v_{3}, u_{2} v_{4} \in E(G)$. According to (1), $u_{p-1} v_{3} \notin E(G)$ and $u_{p-1} v_{4} \notin E(G)$. If there exists $v_{i} \in\left\{v_{1}, v_{2}\right\}$, say $i=1$, such that $v_{1} u_{2} \in E(G)$, then $u_{p-1} v_{1} \notin E(G), N_{H}\left(u_{p-1}\right)=\left\{v_{2}, v_{5}, v_{6}\right\}$ and $u_{p-1} v \in E(G)$. This together with (1) tell us $u_{p}$ has at most one neighbor in $\left\{v_{1}, v_{3}, v_{4}\right\}$ and thus $\left\{v_{2}, v_{5}, v_{6}\right\} \subseteq N_{H}\left(u_{p}\right)$ and $u_{p} v \in E(G)$. We see that $\left[v, u_{p}, u_{p-1}, v_{6}\right] \supseteq K_{4}^{-}$, $\left[u_{1}, u_{2}, v_{3}, v_{4}\right] \supseteq K_{4}^{-}$and $\left[v_{0}, v_{1}, v_{2}, v_{5}\right] \supseteq K_{4}^{-}$, a contradiction. This proves that $u_{2}$ has no neighbor in $\left\{v_{1}, v_{2}\right\}$ and so $u_{2} v_{5}, u_{2} v_{6} \in E(G)$. As $\left|E\left(u_{p-1}, V(H)\right)\right| \geq$ 3 , by the symmetry role of $v_{1}$ and $v_{2}$, we may assume that $v_{1} u_{p-1} \in E(G)$. Suppose that $u_{p-1} v_{6} \in E(G)$. If $v_{6} u_{p} \in E(G)$, then $\left[u_{p-1}, u_{p}, v_{1}, v_{6}\right] \supseteq K_{4}^{-}$, $\left[v, v_{2}, v_{3}, u_{1}\right] \supseteq K_{4}^{-}$and $\left[v_{0}, u_{2}, v_{4}, v_{5}\right] \supseteq K_{4}^{-}$, a contradiction. Therefore, $v_{6} u_{p} \notin E(G)$ and then there exist $v_{i}, v_{j} \in\left\{v_{2}, v_{3}, v_{4}, v_{5}\right\}$, such that $v_{i} u_{p}, v_{j} u_{p} \in$ $E(G)$. If $2 \in\{i, j\}$, then $\left[v, v_{i}, v_{j}, u_{p}\right] \supseteq K_{4}^{-},\left[v_{1}, u_{1}, u_{p-1}, v_{6}\right] \supseteq K_{4}^{-}$and $\left[V\left(H-\left\{v_{1}, v_{i}, v_{j}, v_{6}\right\}\right) \cup\left\{v_{0}, u_{2}\right\}\right] \supseteq K_{4}^{-}$, a contradiction. Hence, $2 \notin\{i, j\}$. Then $\left[u_{2}, v_{i}, v_{j}, u_{p}\right] \supseteq K_{4}^{-},\left[v_{1}, u_{1}, v_{6}, u_{p-1}\right] \supseteq K_{4}^{-}$and $\left[V\left(H-\left\{v_{1}, v_{i}, v_{j}, v_{6}\right\}\right) \cup\right.$ $\left.\left\{v_{0}, v\right\}\right] \supseteq K_{4}^{-}$, a contradiction. This proves that $u_{p-1} v_{6} \notin E(G)$ and it follows that $v_{2} u_{p-1}, v_{5} u_{p-1} \in E(G)$. By $(1), u_{p} v_{5} \notin E(G)$. Since $[V(F-$ $\left.\left.\left\{u_{1}, u_{p}\right\}\right) \cup\left\{v_{5}, v_{6}\right\}\right]$ contains a theta graph and $u_{p}$ has at least two neighbors in $\left\{v_{1}, v_{2}, v_{3}, v_{4}\right\}$, we see that $\left[V\left(H-\left\{v_{5}, v_{6}\right\}\right) \cup\left\{v, u_{1}, u_{p}, v_{0}\right\}\right] \supseteq 2 K_{4}^{-}$, a contradiction. This completes the proof of Case 2 and the proof of Claim 3.5. 
Since $n \geq 13$ and $6 \leq|V(H)| \leq 8$, it follows from Claim 3.5 that $\left|F^{*}\right| \geq 2$.

Claim 3.6. $|V(F)| \leq 2$ for each $F \in F^{*}$.

Proof. By way of contradiction. Suppose that there exists $F \in F^{*}$ such that $|V(F)| \geq 3$. According to Claim 3.5, $|V(F)|=3$. If $F$ is a triangle, then the proof of Claim 3.3 works, because of $\left|F^{*}\right| \geq 2$. Thus, $F$ is a path of order three and write $F=u_{1} u_{4} u_{3}$. Let $F^{\prime} \in F^{*}-F$ and $u_{4} \in V\left(F^{\prime}\right)$ such that $u_{2}$ is an end vertex of $F^{\prime}$. It is obvious that $d_{F^{\prime}}\left(u_{2}\right)=1$. Suppose that $7 \leq l \leq 8$. It is obvious that there exists $v_{i} \in V(H)$, such that $u_{1} v_{i}, u_{4} v_{i}, u_{3} v_{i} \in E(G)$, that is, $\left[v_{i}, u_{1}, u_{4}, u_{3}\right] \supseteq K_{4}^{-}$, since $\left[V\left(H-\left\{v_{i}\right\}\right) \cup\left\{v_{0}, u_{2}\right\}\right] \supseteq 2 K_{4}^{-}$, a contradiction. Thus, $l=6$, then continue the same proof in Claim 3.5 (when $|F| \geq 4$ and contains at least three leaves).

Claim 3.7. For each graph $F \in \mathcal{F}$ such that $|V(F)|=2$, there exists $S \subset V(H)$ with $|S|=2$ and $[V(F) \cup S] \supseteq K_{4}^{-}$.

Proof. Let $F \in \mathcal{F}$ such that $|V(F)|=2$, label $V(F)=\left\{u_{1}, u_{2}\right\}$. Since $\left|E\left(u_{i}, V(H)\right)\right| \geq l-1$ if $7 \leq l \leq 8$ and $\left|E\left(u_{i}, V(H) \cup\{v\}\right)\right| \geq l-1$ for each $i$ with $1 \leq i \leq 2$, it follows from the pigeonhole principle that there exists a subset $S \subset V(H)$ with $|S|=2$ and $S \subseteq N_{H}\left(u_{1}\right) \cap N_{H}\left(u_{2}\right)$. By (3), we know $[V(F) \cup S] \supseteq K_{4}^{-}$.

Claim 3.8. For any $u \in V\left(G^{*}\right),\left|E\left(u,\left\{v_{0}\right\} \cup V(H)\right)\right|=|E(u, V(H))| \leq l-1$ if $7 \leq l \leq 8 ;|E(u, V(H) \cup\{v\})| \leq l$ if $l=6$.

Proof. Suppose that there exists $u \in V\left(G^{*}\right)$ such that $|E(u, V(H))| \geq l$ if $7 \leq l \leq 8$, and $|E(u, V(H) \cup\{v\})| \geq l+1$ if $l=6$. By Claim 3.6, we may assume that $F^{*}$ contains two components $F_{1}$ and $F_{2}$ with $\left|V\left(F_{i}\right)\right| \leq 2$ for each $1 \leq i \leq 2$, such that $u \in V\left(F_{1}\right)$. Suppose that $\left|V\left(F_{2}\right)\right|=2$ and label $F_{2}=u_{2} u_{3}$. Note that $\left|E\left(u_{i}, V(H)\right)\right| \geq l-1$ for each $i \in\{2,3\}$. By Claim 3.7, there exist $v_{i}, v_{j} \in V(H)$ such that $\left[u_{2}, u_{3}, v_{i}, v_{j}\right] \supseteq K_{4}^{-}$. If $7 \leq l \leq 8$, combining with (2) and (3), $\left[V\left(H-\left\{v_{i}, v_{j}\right\}\right) \cup\left\{u, v_{0}\right\}\right] \supseteq 2 K_{4}^{-}$, which contradicts (1). Therefore, $l=6$. By the choice of $v,|E(v, V(H))|=6$. Notice that $v_{p} v_{q} \in E(G)$, thus, $\left[v_{p}, v_{q}, v, u\right] \supseteq K_{4}^{-}$. Since $F^{*} \backslash\left(F_{1} \cup F_{2}\right) \neq \emptyset$, choose $u_{4} \in V\left(F^{*} \backslash\left(F_{1} \cup F_{2}\right)\right)$. By Claim 3.6, $\left|E\left(u_{4}, V(H)\right)\right| \geq 4$, choose $\left\{v_{p}, v_{q}\right\} \subseteq N_{H}\left(u_{4}\right) \cap N_{H}(v)-\left\{v_{i}, v_{j}\right\}$ such that $p \neq q$. Now, $\left[v_{p}, v_{q}, u_{4}, v_{0}\right] \supseteq K_{4}^{-}$and $\left[V\left(H-\left\{v_{i}, v_{j}, v_{p}, v_{q}\right\}\right) \cup\{u, v\}\right] \supseteq$ $K_{4}^{-}$, which contradicts (1). This shows the order of each components of $F^{*} \backslash F_{1}$ is one. Now, note that $\left|F^{*} \backslash F_{1}\right| \geq 3$, we can choose three different vertices $u_{1}, u_{2}, u_{3}$, such that $\left|E\left(u_{i}, V(H)\right)\right| \geq 5$ for each $1 \leq i \leq 3$. As above, it is obvious that $\left[V(H) \cup\left\{v, u, v_{0}, u_{1}, u_{2}, u_{3}\right\}\right] \supseteq 3 K_{4}^{-}$, a contradiction.

Now we are in the position to complete the proof of Theorem 1.8. By Claim 3.6 and Claim 3.8, $|V(F)|=2$ for all $F \in F^{*}$, we have

$$
\sum_{F \in F^{*}}|E(F)|= \begin{cases}\frac{n-1-l}{2}, & \text { if } 7 \leq l \leq 8 \\ \frac{n-8}{2}, & \text { if } l=6 .\end{cases}
$$


Suppose that $7 \leq l \leq 8$. We may assume that $u_{1} u_{2}$ and $u_{3} u_{4}$ are two component of $G^{*}$, since $\left|E\left(u_{i}, V(H)\right)\right| \geq l-1$, by Claim 3.2, it is obvious that $\left[V(H) \cup\left\{v_{0}, u_{1}, u_{2}, u_{3}, u_{4}\right\}\right] \supseteq 3 K_{4}^{-}$, a contradiction. Thus, $l=6$, and according to Claim 3.8, we obtain

$$
\begin{aligned}
|E(G)| & =\left|E\left(\left[\left\{v_{0}, v\right\} \cup V(H)\right]\right)\right|+\left|E\left(V\left(G^{*}\right),\left\{v_{0}, v\right\} \cup V(H)\right)\right|+\sum_{F \in F^{*}}|E(F)| \\
& \leq 27+5\left|V\left(G^{*}\right)\right|+\sum_{F \in F^{*}}|E(F)| \\
& =27+5(n-8)+\frac{n-8}{2} \\
& =\frac{11 n-34}{2},
\end{aligned}
$$

this is an obvious contradiction and completes the proof of Theorem 1.8.

Acknowledgements. The authors wish to thank the referees for their suggestions for revising the manuscript, especially, one of the referees helps us to construct an extremal graph in Conjecture 1.6.

\section{References}

[1] J. A. Bondy and U. S. R. Murty, Graph Theory, the second edition, Springer, 2008.

[2] S. Chiba, S. Fujita, Y. Gao, and G. Li, On a sharp degree sum condition for disjoint chorded cycles in graphs, Graphs Combin. 26 (2010), no. 2, 173-186.

[3] K. Corrádi and A. Hajnal, On the maximal number of independent circuits in a graph, Acta Math. Acad. Sci. Hungar. 14 (1963), 423-439.

[4] G. A. Dirac, On the maximal number of independent triangles, Abh. Math. Sem. Univ. Hamburg 26 (1963), 78-82.

[5] H. Enomoto, On the existence of disjoint cycles in a graph, Combinatorica 18 (1998), no. 4, 487-492.

[6] D. Finkel, On the number Of independent chorded cycle in a graph, Discrete Math. 308 (2008), no. 22, 5265-5268.

[7] Y. Gao and N. Ji, The extremal function for two disjoint cycles, Bull. Malays. Math. Sci. Soc. DOI:10.1007/s40840-014-0102-0, 2014.

[8] K. Kawarabayashi, $K_{4}^{-}$-factor in graphs, J. Graph Theory 39 (2002), no. 2, 111-128.

[9] L. Pósa, On the circuits of finite graphs, Magyar Tud. Akad. Mat. Kut. Int. Közl. 8 (1963), 355-361.

[10] H. Wang, On the maximum number of independent cycles in a graph, Discrete Math. 205 (1999), no. 1-3, 183-190.

Yunshu GaO

School of Mathematics and Computer Science

NingXIA UNIVERSITY

YinCHUAN, 750021, P. R. CHINA

E-mail address: gysh2004@gmail.com 


\section{Ding MA}

School of Mathematics and Computer Science

NingXIA UNIVERSITY

Yinchuan,750021, P. R. China

E-mail address: mading0202@sina.com 